\title{
ON THE WELLPOSEDNESS OF THE CAUCHY PROBLEM FOR WEAKLY HYPERBOLIC EQUATIONS OF HIGHER ORDER
}

\author{
PIERO D'ANCONA AND TAMOTU KINOSHITA
}

\begin{abstract}
We study the wellposedness in the Gevrey classes $G^{s}$ and in $C^{\infty}$ of the Cauchy problem for weakly hyperbolic equations of higher order. In this paper we shall give a new approach to the case that the characteristic roots oscillate rapidly and vanish at an infinite number of points.
\end{abstract}

\section{INTRODUCTION}

We consider here the Cauchy problem on $[0, T] \times \mathbf{R}_{x}^{n}$

$$
\left\{\begin{array}{l}
D_{t}^{m} u=\sum_{j+|\alpha|=m, j<m} c_{j, \alpha}(t) D_{t}^{j} D_{x}^{\alpha} u+\sum_{j+|\alpha| \leq d} c_{j, \alpha}(t) D_{t}^{j} D_{x}^{\alpha} u+f(t, x), \\
D_{t}^{j} u(0, x)=u_{j}(x) \quad(j=0, \cdots, m-1),
\end{array}\right.
$$

where $D_{t}=-i \partial_{t}, D_{x}=-i\left(\partial_{x_{1}}, \cdots, \partial_{x_{n}}\right)$ and $0 \leq d \leq m-1$. We write in short

$$
p(t, \tau, \xi)=\tau^{m}-\sum_{j+|\alpha|=m, j<m} c_{j, \alpha}(t) \tau^{j} \xi^{\alpha}
$$

for the principal part, and

$$
p_{d}(t, \tau, \xi)=\sum_{j+|\alpha| \leq d} c_{j, \alpha}(t) \tau^{j} \xi^{\alpha}
$$

for the lower order terms.

Let $\lambda_{k}(t, \xi)$ be the roots of $p(t, \tau, \xi)=0$ in the sense that

$$
p(t, \tau, \xi)=\prod_{k=1}^{m}\left(\tau-\lambda_{k}(t, \xi)\right) .
$$

We shall assume that the principal part $p$ is hyperbolic with respect to $\tau$, that is, $\lambda_{k}(t, \xi)$ are all real for any $t \in \mathbf{R}_{t}, \xi \in \mathbf{R}_{\xi}^{n}$. Moreover, we name them $\lambda_{k}(t, \xi)$ according to the rule

$$
\lambda_{1}(t, \xi) \geq \lambda_{2}(t, \xi) \geq \cdots \geq \lambda_{m}(t, \xi) .
$$

We recall that the functions $\lambda_{k}(t, \xi)$ are homogeneous of degree 1 in $\xi$.

We denote by $G^{s}\left(\mathbf{R}^{n}\right)$ the space of Gevrey functions $g(x)$ satisfying

$$
\sup _{x \in K}\left|D_{x}^{\alpha} g(x)\right| \leq C_{K} \rho_{K}^{|\alpha|}|\alpha| !^{s} \quad \text { for any compact set } K \subset \mathbf{R}^{n}, \alpha \in \mathbf{N}^{n} .
$$

We say that the Cauchy problem (1) is wellposed in $G^{s}$ (resp. $\left.C^{\infty}\right)$, if for any $u_{j} \in G^{s}\left(\right.$ resp. $\left.C^{\infty}\right)$ and $f \in C^{0}\left([0, T] ; G^{s}\right)\left(\right.$ resp. $\left.C^{0}\left([0, T] ; C^{\infty}\right)\right)$, there is a unique solution $u \in C^{m}\left([0, T] ; G^{s}\right)\left(\right.$ resp. $\left.C^{m}\left([0, T] ; C^{\infty}\right)\right)$. 
There are many results on this problem. As to the wellposedness in $C^{\infty}$, we mention that T. Nishitani [16] considered the case when the multiplicity of characteristic roots is at most double. F. Colombini and N. Orrú [7] assumed that the characteristic roots vanish of finite order at $t=0$ and satisfy

$$
t^{2} \sum_{k, j=1, k \neq j}^{m} \frac{\left|\lambda_{k}^{\prime}(t, \xi)\right|^{2}+\left|\lambda_{j}^{\prime}(t, \xi)\right|^{2}}{\left|\lambda_{k}(t, \xi)-\lambda_{j}(t, \xi)\right|^{2}}<\infty \quad \text { near } t=0 .
$$

Moreover, K. Kajitani, S. Wakabayashi and K. Yagdjian [13] dealt with the case of characteristic roots vanishing of infinite order. Concerning the wellposedness in $G^{s}$, F. Colombini and T. Kinoshita [6] considered the Cauchy problem in the case when characteristic roots are Hölder continuous in $t$. F. Colombini, H. Ishida [4] and H. Ishida, K. Yagdjian [11] assumed that the characteristic roots vanish of infinite order at $t=0$ and satisfy for some $\bar{s}>1$

$$
\frac{\Phi_{1}(t)^{2 \bar{s} /(\bar{s}-1)}}{\phi_{1}(t)^{2}} \sum_{k, j=1, k \neq j}^{m} \frac{\left|\lambda_{k}^{\prime}(t, \xi)\right|^{2}+\left|\lambda_{j}^{\prime}(t, \xi)\right|^{2}}{\left|\lambda_{k}(t, \xi)-\lambda_{j}(t, \xi)\right|^{2}}<\infty \quad \text { near } \quad t=0,
$$

where $\Phi_{1}(t)=\int_{0}^{t} \phi_{1} d t$ and $\phi_{1}(t), \cdots, \phi_{m}(t)$ are real-valued functions such that

(i) $\phi_{k}(0)=\phi_{k}^{\prime}(0)=0, \phi_{k}^{\prime}(t)>0$ if $t \in(0, T]$ for $k=1, \cdots, m$.

(ii) $\phi_{1}(t) \geq \phi_{2}(t) \geq \cdots \geq \phi_{m}(t)$ for $t \in[0, T]$.

(iii) $\left|\lambda_{k}(t, \xi)\right| \leq C_{k} \phi_{k}(t)|\xi|\left({ }^{\exists} C_{k}>0\right)$ for $k=1, \cdots, m,(t, \xi) \in[0, T] \times \mathbf{R}_{\xi}^{n} \backslash 0$.

(iv) $\left|\lambda_{k}(t, \xi)-\lambda_{j}(t, \xi)\right| \geq c \phi_{k}(t)|\xi|\left({ }^{\exists} c>0\right)$ for $k<j,(t, \xi) \in[0, T] \times \mathbf{R}_{\xi}^{n} \backslash 0$.

Then they showed the wellposedness in $G^{s}$ if $1 \leq s<\bar{s}$.

We see that in most results concerning the higher order case $m>2$ the roots are assumed to coincide only at isolated points, and then a precise behavior is assumed at those points. In this paper we try to give a global assumption valid in more general cases, even when this happens at an arbitrary set of points (also infinite or dense). To this end we introduce the sets $\Omega_{\sigma}^{k}, \Omega_{\sigma}$ defined as follows: for any $0<\sigma<1, k=1, \ldots, m-1$,

$$
\Omega_{\sigma}^{k}(\xi)=\left\{t \in[0, T]:\left|\lambda_{k}(t, \xi)-\lambda_{k+1}(t, \xi)\right| \leq \sigma\right\}
$$

and

$$
\Omega_{\sigma}(\xi)=\bigcup_{k=1}^{m-1} \Omega_{\sigma}^{k}(\xi)
$$

These sets enclose, for each $\xi$, the points $t$ where the roots coincide; thus we can regard the measure $\mu\left(\Omega_{\sigma}\right)$, which is a function of $\sigma, \xi$, as a measure of the defect of strict hyperbolicity of $p$. Here $\mu(A)$ is the Lebesgue measure in $\mathbf{R}_{t}$ of the set $A \subseteq[0, T]$.

We denote by $A C([0, T])$ the space of absolutely continuous functions on $[0, T]$.

Our first result is the following:

Theorem 1.1. Assume that the coefficients $c_{j, \alpha}(t)$ of $p_{d}$ belong to $C^{0}([0, T])$ and the characteristic roots of the principal part $\lambda_{1}, \cdots, \lambda_{m}$ belong to $A C([0, T])$ and that there exist constants $C>0, a \geq 0$ and $b>0$ such that

$$
\begin{gathered}
\mu\left(\Omega_{\sigma}(\xi)\right) \leq C \sigma^{a}, \\
\int_{[0, T] \backslash \Omega_{\sigma}^{k}(\xi)} \frac{\left|\lambda_{k}^{\prime}(t, \xi)\right|+\left|\lambda_{k+1}^{\prime}(t, \xi)\right|}{\left|\lambda_{k}(t, \xi)-\lambda_{k+1}(t, \xi)\right|} d t \leq C \sigma^{-b},
\end{gathered}
$$


for any $0<\sigma<1,|\xi|=1, k=1, \cdots, m-1$. Then the Cauchy problem (1) is wellposed in $G^{s}$ if

$$
1 \leq s<\left\{\begin{aligned}
1+\frac{a+1}{b} & \text { when } d \leq \frac{m(a+b)}{a+b+1}, \\
\frac{m}{d+a(d-m)} & \text { when } d>\frac{m(a+b)}{a+b+1} .
\end{aligned}\right.
$$

Remark 1.2. For the simplicity, we assume that the characteristic roots $\lambda_{1}, \cdots, \lambda_{m}$ belong to $A C([0, T])$. If $\mu\left(\Omega_{0}\right)=0$, it is enough that $\lambda_{1}, \cdots, \lambda_{m}$ belong to $A C\left([0, T] \backslash \Omega_{0}\right)$. In particular, in case when $\lambda_{1}, \cdots, \lambda_{m}$ coincide only at $t=0$, we may assume that $\lambda_{1}, \cdots, \lambda_{m}$ belong to $A C((0, T])$.

Remark 1.3. M. D. Bronšhtein [1], S. Wakabayashi [22] and T. Mandai [14] proved the Lipschitz continuity in $t$ of the characteristic roots of hyperbolic polynomials with coefficients in $C^{m}\left([0, T]\right.$ )(see also [20]). If we assume that the coefficients $c_{j, \alpha}$ of $p$ belong to $C^{m}([0, T])$, we can deduce that $\lambda_{1}, \cdots, \lambda_{m}$ belong to $\operatorname{Lip}([0, T])(\subset$ $A C([0, T]))$ and $b=1-a$ in (3) (see Remark 3.3). Thus by Theorem 1.1 the Cauchy problem (1) is wellposed in $G^{s}$ if

$$
1 \leq s< \begin{cases}\frac{2}{1-a} & \text { when } d \leq \frac{m}{2}, \\ \frac{m}{d+a(d-m)} & \text { when } d>\frac{m}{2} .\end{cases}
$$

It is well-known that any lower order term does not influence the wellposedness in $C^{\infty}$ for strictly hyperbolic equations (the multiplicity of characteristic roots is equal to 1) and lower order terms of order $d=m-1$ give the wellposedness in $G^{s}$ if $1 \leq s<m /(m-1)$ for weakly hyperbolic equations (the multiplicity of characteristic roots is equal to $m$ ) (see [1], [2], [3], [5], [10], [18], etc.). As the parameter $a$ in (2) becomes larger, the type of $p$ approaches to strictly hyperbolic type. Especially, when $d=m-1$, by (5) we find that $1 \leq s<m /(m-1-a)$. Taking $0 \leq a<m-1$, we can obtain an interpolation between $C^{\infty}$ and $G^{m /(m-1)}$.

Example 1.4. When the characteristic roots are

$$
\lambda_{k}(t, \xi)=\left\{\begin{array}{cc}
k t^{h}\left\{1+\sin ^{2}\left(\frac{1}{t^{h / \alpha-1}}\right)\right\} \cdot \xi & \text { if } t>0, \\
0 & \text { if } t=0,
\end{array}\right.
$$

for some $0<\alpha \leq 1, h>\alpha$ and $k=1, \cdots, m$, we find that $\lambda_{1}, \cdots, \lambda_{m}$ belong to $A C((0, T])$ (see Remark 1.2) and also to $C^{\alpha}([0, T])$ and vanish of finite order at $t=0$ and satisfy (2) with $a=1 / h$ and (3) with $b=1 / \alpha-1 / h$, since

$$
\begin{aligned}
\mu\left(\Omega_{\sigma}(\xi)\right) & \leq C \int_{0}^{C \sigma^{1 / h}} d t \leq C \sigma^{1 / h}, \\
\int_{[0, T] \backslash \Omega_{\sigma}^{k}(\xi)} \frac{\left|\lambda_{k}^{\prime}(t, \xi)\right|+\left|\lambda_{k+1}^{\prime}(t, \xi)\right|}{\left|\lambda_{k}(t, \xi)-\lambda_{k+1}(t, \xi)\right|} d t & \leq C \int_{C \sigma^{1 / h}}^{T}\left(\frac{-1}{t^{h / \alpha-1}}\right)^{\prime} d t \leq C \sigma^{1 / h-1 / \alpha} .
\end{aligned}
$$

Applying Theorem 1.1, we get the wellposedness in $G^{s}$ if

$$
1 \leq s<\frac{h}{h-\alpha}(1+\alpha)
$$


According to [6] and [18], if characteristic roots belong to $C^{\alpha}([0, T])$ with $0<$ $\alpha \leq 1$, the Cauchy problem (1) is wellposed in $G^{s}$ if

$$
1 \leq s<1+\alpha
$$

For the second order equation $D_{t}^{2} u=A(t) D_{x}^{2} u$ where $A(t) \geq 0$, if $A(t)$ belongs to $C^{2 \alpha}([0, T])$ with $\alpha>0$, we also know the wellposedness in $G^{s}$ under the condition (7) (see [5], [8] and [17]). We remark that (6) approaches to (7) as $h$ tends to infinity and $s$ can be taken arbitrarily large as $h$ tends to $\alpha$ (characteristic roots oscillate more slowly). This example implies that the oscillations and the degeneracy of characteristic roots influence on the wellposedness independently of their regularity.

In general, if the characteristic roots belong to $C^{\alpha}([0, T])$ with $\alpha>1$, we can not expect for higher order equations the wellposedness in $G^{s}$ under the condition (7). But our theorem can be applied into the following:

Example 1.5. When $\lambda_{k}(t, \xi) \equiv k \lambda(t) \cdot \xi$ for $k=1, \cdots, m$, where $\lambda(t) \in C^{\alpha}([0, T])$ with $1 \leq \alpha<\infty$, we find that $\lambda_{1}(t, \xi), \cdots, \lambda_{m}(t, \xi)$ satisfy

$$
\begin{aligned}
& \int_{[0, T] \backslash \Omega_{\sigma}^{k}(\xi)} \frac{\left|\lambda_{k}^{\prime}(t, \xi)\right|+\left|\lambda_{k+1}^{\prime}(t, \xi)\right|}{\left|\lambda_{k}(t, \xi)-\lambda_{k+1}(t, \xi)\right|} d t \leq C \int_{[0, T] \backslash \Omega_{\sigma}^{k}(\xi)} \frac{\left|\lambda^{\prime}(t)\right|}{|\lambda(t)|} d t \\
\leq & C \sigma^{-1 / \alpha} \int_{[0, T] \backslash \Omega_{\sigma}^{k}(\xi)} \frac{\left|\lambda^{\prime}(t)\right|}{|\lambda(t)|^{1-1 / \alpha}} d t \leq C \sigma^{-1 / \alpha} \int_{0}^{T}\left|\left\{\lambda(t)^{1 / \alpha}\right\}^{\prime}\right| d t \leq C \sigma^{-1 / \alpha .}
\end{aligned}
$$

In the last inequality we used Lemma 1 in [5]. Applying Theorem 1.1 with $b=1 / \alpha$ (and $a=0$ ), we get the wellposedness in $G^{s}$ under the condition (7).

Remark 1.6. In the cases mentioned above, when $\lambda_{1}, \cdots, \lambda_{m}$ vanish of infinite order, the assumption (2) can be dropped (one is forced to choose $a=0$ ). Thus by Theorem 1.1 we see that the Cauchy problem (1) is wellposed in $G^{s}$ if

$$
1 \leq s<\min \left\{1+\frac{1}{b}, \frac{m}{d}\right\}
$$

Example 1.7. [4] and [11] gave an example of the following kind:

$$
\lambda_{k}(t, \xi)=\left\{\begin{array}{cc}
k \exp \left(-\frac{1}{t^{h}}\right)\left\{1+\sin ^{2}\left(\exp \frac{\gamma}{t^{h}}\right)\right\} \cdot \xi & \text { if } t>0 \\
0 & \text { if } t=0
\end{array}\right.
$$

for $k=1, \cdots, m$, with some $\gamma>0$ and $h>0$. They proved the wellposedness in $G^{s}$ if $1 \leq s<1+1 / \gamma$. Notice that $\lambda_{1}, \cdots, \lambda_{m}$ belong to $A C((0, T])$ (see Remark 1.2) and vanish of infinite order at $t=0$ (see Remark 1.6) and satisfy (3) with $b=\gamma$;

$$
\int_{[0, T] \backslash \Omega_{\sigma}^{k}(\xi)} \frac{\left|\lambda_{k}^{\prime}(t, \xi)\right|+\left|\lambda_{k+1}^{\prime}(t, \xi)\right|}{\left|\lambda_{k}(t, \xi)-\lambda_{k+1}(t, \xi)\right|} d t \leq C \int_{1 /\left(\log \sigma^{-1}+C\right)^{1 / h}}^{T}\left(-\exp \frac{\gamma}{t^{h}}\right)^{\prime} d t \leq C \sigma^{-\gamma} .
$$

Applying Theorem 1.1, by (8) we get the same Gevrey order $1 \leq s<1+1 / \gamma$.

We consider now the wellposedness in $C^{\infty}$. Our second result is the following:

Theorem 1.8. Assume that the coefficients $c_{j, \alpha}(t)$ of $p_{d}$ belong to $C^{0}([0, T])$ and the characteristic roots of the principal part $\lambda_{1}, \cdots, \lambda_{m}$ belong to $A C([0, T])$ and 
that there exist constants $C>0, a \geq 0$ such that

$$
\mu\left(\Omega_{\sigma}(\xi)\right) \leq C \sigma^{a}
$$

$$
\int_{[0, T] \backslash \Omega_{\sigma}^{k}(\xi)} \frac{\left|\lambda_{k}^{\prime}(t, \xi)\right|+\left|\lambda_{k+1}^{\prime}(t, \xi)\right|}{\left|\lambda_{k}(t, \xi)-\lambda_{k+1}(t, \xi)\right|} d t \leq C \log \sigma^{-1},
$$

for any $0<\sigma<1,|\xi|=1$ and $k=1, \cdots, m-1$. Then, when the degree $d$ of the lower order terms satisfies

$$
0 \leq d \leq \frac{m a}{a+1}
$$

the Cauchy problem (1) is wellposed in $C^{\infty}$.

Remark 1.9. Instead of the assumptions (2) and (9), we assume that there exist constants $C>0, b>0$ such that

$$
\begin{gathered}
\mu\left(\Omega_{\sigma}(\xi)\right) \leq C \exp \left\{-\sigma^{-b}\right\} \\
\int_{[0, T] \backslash \Omega_{\sigma}^{k}(\xi)} \frac{\left|\lambda_{k}^{\prime}(t, \xi)\right|+\left|\lambda_{k+1}^{\prime}(t, \xi)\right|}{\left|\lambda_{k}(t, \xi)-\lambda_{k+1}(t, \xi)\right|} d t \leq C \sigma^{-b},
\end{gathered}
$$

for any $0<\sigma<1,|\xi|=1$ and $k=1, \cdots, m-1$. Then the Cauchy problem (1) is wellposed in $C^{\infty}$ for any lower order terms.

Example 1.10. Let $B(t)$ be a positive $C^{1}$ function and $\lambda(t)$ be a smooth function such that $\lambda(0)=\lambda^{\prime}(0)=0$ and $\lambda(t)>0, \lambda^{\prime}(t) \geq 0$ for all $t>0$. We shall suppose that $B(t)$ and $\lambda(t)$ satisfy

$$
\left|D_{t} B(t)\right| \leq C \frac{\lambda^{\prime}(t)}{\lambda(t)} \text { for } t>0
$$

For the second order equation

$$
D_{t}^{2} u=\lambda(t)^{2} B(t)^{2} D_{x}^{2} u
$$

the wellposedness in $C^{\infty}$ can be easily shown under the condition (11). (see [7], [13], [23], etc.). While, by (11) we find that $\lambda_{1}, \lambda_{2}$ belong to $A C([0, T])$ and satisfy

$$
\int_{[0, T] \backslash \Omega_{\sigma}^{k}(\xi)} \frac{\left|\lambda_{1}^{\prime}(t, \xi)\right|+\left|\lambda_{2}^{\prime}(t, \xi)\right|}{\left|\lambda_{1}(t, \xi)-\lambda_{2}(t, \xi)\right|} d t \leq C \int_{\lambda^{-1}(\sigma)}^{T} \frac{\lambda^{\prime}(t)}{\lambda(t)} d t \leq C \log \sigma^{-1} .
$$

Therefore, we can also apply Theorem 1.8 and get the wellposedness in $C^{\infty}$. In fact, M. Reissig, K. Yagdjian [19] and S. Tarama [21] proved for the 2nd order equation (12) the wellposedness in $C^{\infty}$ under the more relaxed condition

$$
\left|D_{t}^{j} B(t)\right| \leq C\left(\frac{\lambda^{\prime}(t)}{\lambda(t)}|\log \lambda(t)|\right)^{j} \text { for } t>0 \text { and } j=1,2 .
$$

Our theorems can be applied also when the vanishing order of characteristic roots is different from the order of contact between the roots. For instance, if the characteristic polynomial is

$$
p(t, \tau, \xi)=\tau^{2}-2 t^{\alpha} \tau \xi+\left(t^{2 \alpha}-t^{2 \beta}\right) \xi^{2} \text { where } 0<\alpha \leq \beta,
$$

we easily obtain $\lambda_{1}(t, \xi)=\left(t^{\alpha}+t^{\beta}\right) \xi$ and $\lambda_{2}(t, \xi)=\left(t^{\alpha}-t^{\beta}\right) \xi$ which implies that $\left|\lambda_{k}(t, \xi)\right| \leq 2 t^{\alpha}|\xi|(k=1,2),\left|\lambda_{1}(t, \xi)-\lambda_{2}(t, \xi)\right| \geq 2 t^{\beta}|\xi|$ for $(t, \xi) \in[0, T] \times \mathbf{R}_{\xi}$. Since $\lambda_{1}(t, \xi)$ and $\lambda_{2}(t, \xi)$ satisfy (2) with $a=1 / \beta$ and (3) with $b=1-\alpha / \beta$, applying 
Theorem 1.1 and Theorem 1.8 we get the wellposedness in $C^{\infty}$ when $\alpha=\beta$, while when $\alpha<\beta$ we have wellposedness in $G^{s}$ if

$$
1 \leq s<1+\frac{\beta+1}{\beta-\alpha} .
$$

We note that the assumptions (2) and (3) can be simplified in the favorable case of analytic characteristic roots. The following corollaries are an immediate consequence of Theorem 1.1 and Theorem 1.8 respectively:

Corollary 1.11. Assume that the coefficients $c_{j, \alpha}(t)$ of $p_{d}$ belong to $C^{0}([0, T])$ and the characteristic roots of the principal part $\lambda_{1}, \cdots, \lambda_{m}$ are analytic in $t$ and vanish at $t=0$ and that there exist constants $C>0, c>0$ and $0<\alpha<\beta$ such that

$$
\begin{gathered}
\left|\lambda_{k}(t, \xi)\right| \leq C t^{\alpha}|\xi| \text { for } k=1, \cdots, m, \\
\left|\lambda_{k+1}(t, \xi)-\lambda_{k}(t, \xi)\right| \geq c t^{\beta}|\xi| \text { for } k=1, \cdots, m-1,
\end{gathered}
$$

for any $(t, \xi) \in[0, T] \times \mathbf{R}_{\xi}^{n}$. Then the Cauchy problem (1) is wellposed in $G^{s}$ if

$$
1 \leq s< \begin{cases}1+\frac{\beta+1}{\beta-\alpha} & \text { when } d \leq \frac{m(\beta-\alpha+1)}{2 \beta-\alpha+1} \\ \frac{\beta m}{\beta d+d-m} & \text { when } d>\frac{m(\beta-\alpha+1)}{2 \beta-\alpha+1}\end{cases}
$$

Corollary 1.12. Assume that the coefficients $c_{j, \alpha}(t)$ of $p_{d}$ belong to $C^{0}([0, T])$ and the characteristic roots of the principal part $\lambda_{1}, \cdots, \lambda_{m}$ are analytic in $t$ and vanish at $t=0$ and that there exists $C>0$ such that

$$
\left|\lambda_{k}(t, \xi)\right|+\left|\lambda_{k+1}(t, \xi)\right| \leq C\left|\lambda_{k+1}(t, \xi)-\lambda_{k}(t, \xi)\right|
$$

for any $(t, \xi) \in[0, T] \times \mathbf{R}_{\xi}^{n}$. Then the Cauchy problem (1) is wellposed in $C^{\infty}$ when the degree $d$ of the lower order terms satisfies

$$
0 \leq d \leq \frac{m}{\beta+1}
$$

where $\beta>0$ is the smallest number such that

$$
\left|\lambda_{k+1}(t, \xi)-\lambda_{k}(t, \xi)\right| \geq c t^{\beta}|\xi| \text { for any }(t, \xi) \in[0, T] \times \mathbf{R}_{\xi}^{n} \text { and } k=1, \cdots, m-1 \text {. }
$$

Remark 1.13. For the second order equation $D_{t}^{2} u=A(t) D_{x}^{2} u$ where $A(t) \geq 0$, we can drop (13). Thus, we get the wellposedness in $C^{\infty}$ under the only assumption that characteristic roots are analytic in $t$ (see [5], [9]).

Remark 1.14. For the second order equation $D_{t}^{2} u=t^{2 \beta} D_{x}^{2} u+D_{x} u$, it is known that $0 \leq \beta \leq 1$ is necessary and sufficient for the wellposedness in $C^{\infty}$ (see [12]). Applying Corollary 1.12 with $m=2$ and $d=1$, we also get the wellposedness in $C^{\infty}$ if $0 \leq \beta \leq 1$.

In Corollaries 1.11, 1.12 and Examples 1.4, 1.7, 1.10, characteristic roots coincide only at $t=0$ or at a finite number of points. We give a final example to emphasize that our results allow characteristic roots to coincide at an infinite number of points. 
Example 1.15 (see also Example 1.4 with $\alpha=1$ ). When the characteristic roots are

$$
\lambda_{k}(t, \xi)=\left\{\begin{array}{cc}
k t^{h} \sin ^{h}\left(\frac{1}{t^{h-1}}\right) \cdot \xi & \text { if } t>0, \\
0 & \text { if } t=0,
\end{array}\right.
$$

for some even number $h$ and $k=1, \cdots, m$, we find that $\lambda_{1}, \cdots, \lambda_{m}$ belong to $A C([0, T])$ and also $\operatorname{Lip}([0, T])$ and vanish at $=(\pi j)^{1 /(1-h)}(j=1,2, \cdots)$ and satisfy (2) with $a<1 / h$ and (3) with $b>1-1 / h$ (see the Appendix). Applying Theorem 1.1, we get the wellposedness in $G^{s}$ if $1 \leq s<2 h /(h-1)$ (compare with (7)).

\section{Estimates for Hyperbolic Operators}

When $s=1$, the Cauchy problem (1) is wellposed in the class of real analytic functions. Therefore we can suppose that $s>1$ for the proof. By Fourier transform with respect to $x$, the Cauchy problem (1) turns into

$$
\left\{\begin{array}{l}
p\left(t, D_{t}, \xi\right) \hat{u}=\hat{f}_{1}(t, \xi), \\
D_{t}^{j} \hat{u}(0, \xi)=\hat{u}_{j}(\xi) \quad(j=0, \cdots, m-1),
\end{array}\right.
$$

where we are using the notation

$$
f_{1}=f+p_{d}\left(t, D_{t}, D_{x}\right) u
$$

i.e., we put the lower order terms in $f$ for the moment.

Let $0<\sigma<1$ and $\varphi(r)$ be a non-negative function such that $\varphi \in C_{0}^{\infty}(\mathbf{R})$, $\varphi(r) \equiv 0$ for $|r| \geq 2$ and $\varphi(r) \equiv 1$ for $|r| \leq 1$. We define

$$
\begin{aligned}
& \omega(t, \xi)=\sigma|\xi| \sum_{l=1}^{m-1} \varphi\left(\sigma^{-1}\left\{\lambda_{l}\left(t, \frac{\xi}{|\xi|}\right)-\lambda_{l+1}\left(t, \frac{\xi}{|\xi|}\right)\right\}\right), \\
& \mu_{k}(t, \xi)=\lambda_{k}(t, \xi)+i k \omega(t, \xi) \text { for } k=1, \cdots, m .
\end{aligned}
$$

Here we remark that $\omega(t, \xi) \geq \sigma|\xi|$ for any $(t, \xi) \in \Omega_{\sigma}(\xi) \times \mathbf{R}_{\xi}^{n} \backslash 0$, and also that for any $(t, \xi) \in[0, T] \times \mathbf{R}_{\xi}^{n} \backslash 0$

$$
\min _{1 \leq k \leq m-1}\left|\mu_{k}(t, \xi)-\mu_{k+1}(t, \xi)\right| \equiv \min _{1 \leq k \leq m-1}\left\{\left|\lambda_{k}(t, \xi)-\lambda_{k+1}(t, \xi)\right|+\omega(t, \xi)\right\} \geq \sigma|\xi| .
$$

Moreover we denote by $q(t, \tau, \xi)$ the polynomial of degree $m$ in $\tau$

$$
q(t, \tau, \xi)=\prod_{k=1}^{m}\left(\tau-\mu_{k}(t, \xi)\right)
$$

Now we set the energy density

$$
E(t, \xi)=\frac{1}{2} \sum_{l=1}^{m}\left|q_{l}\left(t, D_{t}, \xi\right) \hat{u}\right|^{2},
$$

where $q_{l}(t, \tau, \xi)$ is the polynomial of degree $m-1$ in $\tau$ defined by

$$
q_{l}(t, \tau, \xi)=\frac{q(t, \tau, \xi)}{\tau-\mu_{l}(t, \xi)}\left(=\prod_{k=1, k \neq l}^{m}\left(\tau-\mu_{k}(t, \xi)\right)\right) .
$$


There exists $C>0$ such that for any $(t, \xi) \in[0, T] \times \mathbf{R}_{\xi}^{n} \backslash 0$

$$
\sqrt{E(t, \xi)} \leq C \sum_{j=0}^{m-1}|\xi|^{m-1-j}\left|D_{t}^{j} \hat{u}\right| .
$$

On the other hand, we need the following well-known formula in order to show the boundness from below:

Lemma 2.1 (Newton-Lagrange Interpolation Formula). Let $\mu_{1}, \cdots, \mu_{m}$ be distinct. Then any polynomial $g(\tau)$ of degree $(m-1)$ can be written as follows:

$$
g(\tau)=\sum_{l=1}^{m} \prod_{k=1, k \neq l}^{m} \frac{\tau-\mu_{k}}{\mu_{l}-\mu_{k}} g\left(\mu_{l}\right) .
$$

Putting $g(\tau) \equiv \tau^{j}$ in (16), we obtain for $j=0, \cdots, m-1$

$$
\tau^{j} \equiv \sum_{l=1}^{m} \prod_{k=1, k \neq l}^{m} \frac{\tau-\mu_{k}(t, \xi)}{\mu_{l}(t, \xi)-\mu_{k}(t, \xi)} \mu_{l}(t, \xi)^{j}=\sum_{l=1}^{m} \frac{q_{l}(t, \tau, \xi)}{\prod_{k=1, k \neq l}^{m}\left(\mu_{l}(t, \xi)-\mu_{k}(t, \xi)\right)} \mu_{l}(t, \xi)^{j} .
$$

Hence we get

$$
\begin{aligned}
\left|D_{t}^{j} \hat{u}\right|^{2} & =\sum_{l=1}^{m}\left[\frac{\mu_{l}(t, \xi)^{j} q_{l}\left(t, D_{t}, \xi\right) \hat{u}}{\prod_{k=1, k \neq l}^{m}\left(\mu_{l}(t, \xi)-\mu_{k}(t, \xi)\right)} \cdot \overline{D_{t}^{j} \hat{u}}\right] \\
& \leq C|\xi|^{j} \frac{\left\{\sum_{l=1}^{m}\left|q_{l}\left(t, D_{t}, \xi\right) \hat{u}\right|^{2}\right\}^{\frac{1}{2}}\left|D_{t}^{j} \hat{u}\right|}{\max _{1 \leq l \leq m} \prod_{k=1, k \neq l}^{m}\left|\mu_{l}(t, \xi)-\mu_{k}(t, \xi)\right|} .
\end{aligned}
$$

Thus, there exists $c>0$ such that for any $(t, \xi) \in[0, T] \times \mathbf{R}_{\xi}^{n} \backslash 0$

$$
\sqrt{E(t, \xi)} \geq c(\sigma|\xi|)^{m-1}|\xi|^{-j}\left|D_{t}^{j} \hat{u}\right| \text { for } j=0, \cdots, m-1 .
$$

We denote by ' the derivative in $t$. Differentiating $E(t, \xi)$ in $t$, we have

$$
\begin{aligned}
E^{\prime}(t, \xi) & =-\sum_{j=1}^{m} \Im\left[D_{t}\left\{q_{j}\left(t, D_{t}, \xi\right) \hat{u}\right\} \cdot \overline{q_{j}\left(t, D_{t}, \xi\right) \hat{u}}\right] \\
& =-\sum_{j=1}^{m} \Im\left[\left\{-i q_{j}^{\prime}\left(t, D_{t}, \xi\right)+q\left(t, D_{t}, \xi\right)+\mu_{j}(t, \xi) q_{j}\left(t, D_{t}, \xi\right)\right\} \hat{u}\right. \\
& \leq \sum_{j=1}^{m} \Re\left[q_{j}^{\prime}\left(t, D_{t}, \xi\right) \hat{u} \cdot \overline{q_{j}\left(t, D_{t}, \xi\right) \hat{u}}\right] \\
& -\sum_{j=1}^{m} \Im\left[q\left(t, D_{t}, \xi\right) \hat{u}\right]
\end{aligned}
$$

In order to estimate the first and second terms, we shall use again Lemma 1.11. 
2.1. Estimate of the First Term. Putting $g(\tau) \equiv q_{j}^{\prime}(t, \tau, \xi)$ in (16), we obtain

$$
\begin{aligned}
q_{j}^{\prime}(t, \tau, \xi) & \equiv \sum_{l=1}^{m} \prod_{k=1, k \neq l}^{m} \frac{\tau-\mu_{k}}{\mu_{l}-\mu_{k}} q_{j}^{\prime}\left(t, \mu_{l}, \xi\right)=\sum_{l=1}^{m} \frac{q_{l}(t, \tau, \xi)}{q_{l}\left(t, \mu_{l}, \xi\right)} \sum_{k=1, k \neq j}^{m} \frac{-\mu_{k}^{\prime} q_{j}\left(t, \mu_{l}, \xi\right)}{\mu_{l}-\mu_{k}} \\
& =q_{j}(t, \tau, \xi) \sum_{k=1, k \neq j}^{m} \frac{-\mu_{k}^{\prime}}{\mu_{j}-\mu_{k}}+\sum_{l=1, l \neq j}^{m} q_{l}(t, \tau, \xi) \frac{-\mu_{l}^{\prime}}{\mu_{l}-\mu_{j}} \\
& =\sum_{k=1, k \neq j}^{m} \frac{-\mu_{k}^{\prime}}{\mu_{k}-\mu_{j}}\left\{q_{j}(t, \tau, \xi)-q_{k}(t, \tau, \xi)\right\} .
\end{aligned}
$$

Hence we get

$$
\begin{aligned}
& \sum_{j=1}^{m} \Re\left[q_{j}^{\prime}\left(t, D_{t}, \xi\right) \hat{u} \cdot \overline{q_{j}\left(t, D_{t}, \xi\right) \hat{u}}\right] \\
= & \sum_{j=1}^{m} \sum_{k=1, k \neq j}^{m} \Re\left[\frac{-\mu_{k}^{\prime}}{\mu_{j}-\mu_{k}}\left\{q_{j}\left(t, D_{t}, \xi\right)-q_{k}\left(t, D_{t}, \xi\right)\right\} \hat{u} \cdot \overline{q_{j}\left(t, D_{t}, \xi\right) \hat{u}}\right] \\
(20) \leq & C \sum_{j, k=1, j \neq k}^{m} \frac{\left|\mu_{k}^{\prime}\right|}{\left|\mu_{k}-\mu_{j}\right|} E(t, \xi) \leq C \max _{1 \leq k \leq m-1} \frac{\left|\lambda_{k}^{\prime}\right|+\left|\lambda_{k+1}^{\prime}\right|+\left|\omega^{\prime}\right|}{\left|\lambda_{k}-\lambda_{k+1}\right|+\omega} E(t, \xi) .
\end{aligned}
$$

2.2. Estimate of the Second Term. Since $q(t, \tau, \xi)-p(t, \tau, \xi)$ is a polynomial of degree $(m-1)$ in $\tau$, putting $g(\tau) \equiv q(t, \tau, \xi)-p(t, \tau, \xi)$ in $(16)$, we obtain

$$
\begin{aligned}
q(t, \tau, \xi)-p(t, \tau, \xi) & \equiv \sum_{l=1}^{m} \prod_{k=1, k \neq l}^{m} \frac{\tau-\mu_{k}}{\mu_{l}-\mu_{k}}\left\{q\left(t, \mu_{l}, \xi\right)-p\left(t, \mu_{l}, \xi\right)\right\} \\
& =-\sum_{l=1}^{m} \frac{q_{l}(t, \tau, \xi)}{q_{l}\left(t, \mu_{l}, \xi\right)} p\left(t, \mu_{l}, \xi\right) \\
& =-\sum_{l=1}^{m} \prod_{k=1, k \neq l}^{m} \frac{\mu_{l}-\lambda_{k}}{\mu_{l}-\mu_{k}}\left\{\mu_{l}-\lambda_{l}\right\} q_{l}(t, \tau, \xi),
\end{aligned}
$$

here we used $q\left(t, \mu_{l}, \xi\right)=0$. Hence we get

$$
\begin{aligned}
& -\sum_{j=1}^{m} \Im\left[\left\{q\left(t, D_{t}, \xi\right)-p\left(t, D_{t}, \xi\right)\right\} \hat{u} \cdot \overline{q_{j}\left(t, D_{t}, \xi\right) \hat{u}}\right] \\
= & \sum_{j=1}^{m} \sum_{l=1}^{m} \Im\left[\prod_{k=1, k \neq l}^{m} \frac{\mu_{l}-\lambda_{k}}{\mu_{l}-\mu_{k}}\left\{\mu_{l}-\lambda_{l}\right\} q_{l}\left(t, D_{t}, \xi\right) \hat{u} \cdot \overline{q_{j}\left(t, D_{t}, \xi\right) \hat{u}}\right] \\
= & \sum_{j=1}^{m} \sum_{l=1}^{m} \Im\left[\prod_{k=1, k \neq l}^{m}\left\{1+\frac{i k \omega}{\lambda_{l}-\lambda_{k}+i(l-k) \omega}\right\} i l \omega q_{l}\left(t, D_{t}, \xi\right) \hat{u} \cdot \overline{q_{j}\left(t, D_{t}, \xi\right) \hat{u}}\right] \\
\leq & \sum_{j=1}^{m} \sum_{l=1}^{m} \prod_{k=1, k \neq l}^{m}(1+k) l \omega\left|q_{l}\left(t, D_{t}, \xi\right) \hat{u} \cdot \overline{q_{j}\left(t, D_{t}, \xi\right) \hat{u}}\right| \leq C \omega E(t, \xi) .
\end{aligned}
$$


Therefore, by (14) it follows that

$$
\begin{aligned}
& -\sum_{j=1}^{m} \Im\left[q\left(t, D_{t}, \xi\right) \hat{u} \cdot \overline{q_{j}\left(t, D_{t}, \xi\right) \hat{u}}\right] \\
= & -\sum_{j=1}^{m} \Im\left[\left\{q\left(t, D_{t}, \xi\right)-p\left(t, D_{t}, \xi\right)\right\} \hat{u} \cdot \overline{q_{j}\left(t, D_{t}, \xi\right) \hat{u}}\right]-\sum_{j=1}^{m} \Im\left[p\left(t, D_{t}, \xi\right) \hat{u} \cdot \overline{q_{j}\left(t, D_{t}, \xi\right) \hat{u}}\right] \\
\leq & C \omega E(t, \xi)-\sum_{j=1}^{m} \Im\left[\hat{f}_{1}(t, \xi) \cdot \overline{q_{j}\left(t, D_{t}, \xi\right) \hat{u}}\right] \leq C \omega E(t, \xi)+\left|\hat{f}_{1}(t, \xi)\right| \sqrt{E(t, \xi)} .
\end{aligned}
$$

We now recall that $f_{1}=f+p_{d} u$. Since $p_{d}$ has degree $d$, recalling (17) we can write

$$
\begin{aligned}
\left|p_{d}\left(t, D_{t}, \xi\right) \hat{u}\right| & \leq C \sum_{j=0}^{d}|\xi|^{d-j}\left|D_{t}^{j} \hat{u}\right| \leq C \sum_{j=0}^{d}|\xi|^{d-j} \frac{|\xi|^{j}\left\{\sum_{l=1}^{m}\left|q_{l}\left(t, D_{t}, \xi\right) \hat{u}\right|^{2}\right\}^{\frac{1}{2}}}{\max _{1 \leq l \leq m} \prod_{k=1, k \neq l}^{m}\left|\mu_{l}(t, \xi)-\mu_{k}(t, \xi)\right|} \\
& \leq C \frac{|\xi|^{d} \sqrt{E(t, \xi)}}{\prod_{k=1}^{m-1}\left|\lambda_{k}(t, \xi)-\lambda_{k+1}(t, \xi)\right|+\omega(t, \xi)^{m-1}}
\end{aligned}
$$

In conclusion,

$$
\begin{aligned}
-\sum_{j=1}^{m} \Im\left[q\left(t, D_{t}, \xi\right) \hat{u} \cdot \overline{q_{j}\left(t, D_{t}, \xi\right) \hat{u}}\right] \leq & C \omega E(t, \xi)+|\hat{f}(t, \xi)| \sqrt{E(t, \xi)} \\
& +\frac{C|\xi|^{d} E(t, \xi)}{\prod_{k=1}^{m-1}\left|\lambda_{k}-\lambda_{k+1}\right|+\omega^{m-1}}
\end{aligned}
$$

2.3. Complete Energy Estimate. Dividing (19), (20) and (21) by $2 \sqrt{E(t, \xi)}$, we have

$\sqrt{E}^{\prime} \leq C\left(\max _{1 \leq k \leq m-1} \frac{\left|\lambda_{k}^{\prime}\right|+\left|\lambda_{k+1}^{\prime}\right|+\left|\omega^{\prime}\right|}{\left|\lambda_{k}-\lambda_{k+1}\right|+\omega}+\omega+\frac{|\xi|^{d}}{\prod_{k=1}^{m-1}\left|\lambda_{k}-\lambda_{k+1}\right|+\omega^{m-1}}\right) \sqrt{E}+|\hat{f}|$.

Thus, Gronwall's inequality yields the estimate

$$
\begin{gathered}
\sqrt{E(t, \xi)} \leq \exp \left\{C \int_{0}^{T}\left(\max _{1 \leq k \leq m-1} \frac{\left|\lambda_{k}^{\prime}\right|+\left|\lambda_{k+1}^{\prime}\right|+\left|\omega^{\prime}\right|}{\left|\lambda_{k}-\lambda_{k+1}\right|+\omega}+\omega+\frac{|\xi|^{d}}{\prod_{k=1}^{m-1}\left|\lambda_{k}-\lambda_{k+1}\right|+\omega^{m-1}}\right) d t\right\} \\
\times\left\{\sqrt{E(0, \xi)}+\int_{0}^{T}|\hat{f}(t, \xi)| d t\right\} .
\end{gathered}
$$

Moreover, taking (15) and (18) into consideration, we finally obtain the estimate

$$
\begin{aligned}
\sum_{j=0}^{m-1}|\xi|^{-j}\left|D_{t}^{j} \hat{u}(t, \xi)\right| \leq & C \sigma^{1-m} \exp \left\{C \sum_{\nu=1}^{4} \int_{0}^{T} M_{\nu}(t, \xi) d t\right\} \\
& \times\left\{\sum_{j=0}^{m-1}|\xi|^{-j}\left|\hat{u}_{j}(\xi)\right|+\int_{0}^{T}|\xi|^{1-m}|\hat{f}(t, \xi)| d t\right\}
\end{aligned}
$$


where

$$
\begin{aligned}
M_{1}(t, \xi) & =\max _{1 \leq k \leq m-1} \frac{\left|\lambda_{k}^{\prime}(t, \xi)\right|+\left|\lambda_{k+1}^{\prime}(t, \xi)\right|}{\left|\lambda_{k}(t, \xi)-\lambda_{k+1}(t, \xi)\right|+\omega(t, \xi)} \\
M_{2}(t, \xi) & =\max _{1 \leq k \leq m-1} \frac{\left|\omega^{\prime}(t, \xi)\right|}{\left|\lambda_{k}(t, \xi)-\lambda_{k+1}(t, \xi)\right|+\omega(t, \xi)} \\
M_{3}(t, \xi) & =\omega(t, \xi), \quad M_{4}(t, \xi)=\frac{|\xi|^{d}}{\prod_{k=1}^{m-1}\left|\lambda_{k}(t, \xi)-\lambda_{k+1}(t, \xi)\right|+\omega(t, \xi)^{m-1}}
\end{aligned}
$$

\section{Proof of Theorem 1}

Based on the estimate (22), with assumptions (2) and (3) we shall derive an a-priori estimate which shows the wellposedness in Gevrey classes of order (4). In order to estimate $\int_{0}^{T} M_{\nu}(t, \xi) d t$, we need the following statements:

Lemma 3.1. Let $b \geq 0$. Assume that $\lambda_{1}, \cdots, \lambda_{m}$ belong to $A C([0, T])$ and satisfy (3). Then there exists $C>0$ such that for any $0<\sigma<1,|\xi|=1$ and $k=1, \cdots, m$

$$
\int_{\Omega_{\sigma}^{k}(\xi) \cup \Omega_{\sigma}^{k-1}(\xi)}\left|\lambda_{k}^{\prime}(t, \xi)\right| d t \leq\left\{\begin{array}{ll}
C & \text { if } b \geq 1 \\
C \sigma^{1-b} & \text { if } 0 \leq b<1
\end{array} \leq C \sigma^{1-b},\right.
$$

where $\Omega_{\sigma}^{0}(\xi)=\Omega_{\sigma}^{m}(\xi)=\phi$ and $\Omega_{\sigma}^{k}(\xi)$ for $k=1, \cdots, m-1$ are defined in $\S .1$.

Proof. When $b \geq 1$, we immediately obtain (23), since $\lambda_{1}, \cdots, \lambda_{m}$ belong to $A C([0, T])$. For a fixed $k$, we find that $\Omega_{\sigma / 2^{0}}^{k} \supset \Omega_{\sigma / 2^{1}}^{k} \supset \cdots \supset \Omega_{\sigma / 2^{j}}^{k} \supset \cdots$. Let us denote that $\Psi_{j}^{k}=\Omega_{\sigma / 2^{j}}^{k} \backslash \Omega_{\sigma / 2^{j+1}}^{k}$. When $0 \leq b<1$, by (3) we obtain for $|\xi|=1$

$$
\begin{aligned}
\int_{\Omega_{\sigma}^{k} \cup \Omega_{\sigma}^{k-1}}\left|\lambda_{k}^{\prime}\right| d t & \leq \int_{\Omega_{\sigma}^{k}}\left|\lambda_{k}^{\prime}\right| d t+\int_{\Omega_{\sigma}^{k-1}}\left|\lambda_{k}^{\prime}\right| d t \leq \sum_{j=0}^{\infty} \int_{\Psi_{j}^{k}}\left|\lambda_{k}^{\prime}\right| d t+\sum_{j=0}^{\infty} \int_{\Psi_{j}^{k-1}}\left|\lambda_{k}^{\prime}\right| d t \\
& =\sum_{j=0}^{\infty} \int_{\Psi_{j}^{k}} \frac{\left|\lambda_{k}^{\prime}\right|}{\left|\lambda_{k}-\lambda_{k+1}\right|}\left|\lambda_{k}-\lambda_{k+1}\right| d t+\sum_{j=0}^{\infty} \int_{\Psi_{j}^{k-1}} \frac{\left|\lambda_{k}^{\prime}\right|}{\left|\lambda_{k-1}-\lambda_{k}\right|}\left|\lambda_{k-1}-\lambda_{k}\right| d t \\
& \leq \sum_{j=0}^{\infty} \frac{\sigma}{2^{j}} \int_{\Psi_{j}^{k}} \frac{\left|\lambda_{k}^{\prime}\right|}{\left|\lambda_{k}-\lambda_{k+1}\right|} d t+\sum_{j=0}^{\infty} \frac{\sigma}{2^{j}} \int_{\Psi_{j}^{k-1}} \frac{\left|\lambda_{k}^{\prime}\right|}{\left|\lambda_{k-1}-\lambda_{k}\right|} d t \\
& \leq 2 \sum_{j=0}^{\infty} \frac{\sigma}{2^{j}} \cdot C\left(\frac{\sigma}{2^{j+1}}\right)^{-b}=2^{1+b} C \sigma^{1-b} \sum_{j=0}^{\infty} \frac{1}{2^{j(1-b)}} \leq C \sigma^{1-b} .
\end{aligned}
$$

Lemma 3.2. Let $0 \leq a<m-1$. Assume that $\lambda_{1}, \cdots, \lambda_{m}$ satisfy (2). Then there exists $C>0$ such that for any $0<\sigma<1,|\xi|=1$

$$
\int_{[0, T] \backslash \Omega_{\sigma}(\xi)} \frac{d t}{\prod_{k=1}^{m-1}\left|\lambda_{k}(t, \xi)-\lambda_{k+1}(t, \xi)\right|} \leq C \sigma^{a+1-m},
$$

where $\Omega_{\sigma}(\xi)$ is defined in $\S .1$.

Remark 3.3. Let $0 \leq a<1$. When $\lambda_{1}, \cdots, \lambda_{m}$ belong to $\operatorname{Lip}([0, T])$, we also obtain $b=1-a$ in (3), since for any $0<\sigma<1,|\xi|=1$

$$
\int_{[0, T] \backslash \Omega_{\sigma}^{k}(\xi)} \frac{\left|\lambda_{k}^{\prime}(t, \xi)\right|+\left|\lambda_{k+1}^{\prime}(t, \xi)\right|}{\left|\lambda_{k}(t, \xi)-\lambda_{k+1}(t, \xi)\right|} d t \leq C \int_{[0, T] \backslash \Omega_{\sigma}^{k}(\xi)} \frac{d t}{\left|\lambda_{k}(t, \xi)-\lambda_{k+1}(t, \xi)\right|} \leq C \sigma^{a-1} .
$$


Proof. Let $J=J(\sigma)$ be the largest number such that

$$
2^{J} \sigma \leq \max _{1 \leq k \leq m-1,0 \leq t \leq T,|\xi|=1}\left|\lambda_{k}(t, \xi)-\lambda_{k+1}(t, \xi)\right| \equiv \text { const. }
$$

Noting that $\Omega_{2^{0} \sigma} \subset \Omega_{2^{1} \sigma} \subset \cdots \subset \Omega_{2^{J} \sigma} \subset[0, T]$ and denoting that $\Phi_{j}=\Omega_{2^{j+1} \sigma} \backslash \Omega_{2^{j} \sigma}$, by (2) we obtain for $|\xi|=1$

$$
\begin{aligned}
\int_{[0, T] \backslash \Omega_{\sigma}} \frac{d t}{\prod_{k=1}^{m-1}\left|\lambda_{k}-\lambda_{k+1}\right|} & \leq \sum_{j=0}^{J} \int_{\Phi_{j}} \frac{d t}{\prod_{k=1}^{m-1}\left|\lambda_{k}-\lambda_{k+1}\right|} \leq \sum_{j=0}^{\infty} \frac{1}{\left(2^{j} \sigma\right)^{m-1}} \int_{\Phi_{j}} d t \\
& \leq \sum_{j=0}^{\infty} \frac{1}{\left(2^{j} \sigma\right)^{m-1}} \mu\left(\Omega_{2^{j+1} \sigma}\right) \leq \sum_{j=0}^{\infty} \frac{C\left(2^{j+1} \sigma\right)^{a}}{\left(2^{j} \sigma\right)^{m-1}} \\
& =2^{a} C \sigma^{a+1-m} \sum_{j=0}^{\infty} \frac{1}{2^{j(m-1-a)}} \leq C \sigma^{a+1-m}
\end{aligned}
$$

Since $\lambda_{1}(t, \xi), \cdots, \lambda_{m}(t, \xi)$ are homogeneous of degree 1 in $\xi$, by (3) and (23) we get

$$
\begin{aligned}
\int_{0}^{T} M_{1}(t, \xi) d t= & \int_{0}^{T} \max _{1 \leq k \leq m-1} \frac{\left|\lambda_{k}^{\prime}(t, \xi /|\xi|)\right|+\left|\lambda_{k+1}^{\prime}(t, \xi /|\xi|)\right|}{\left|\lambda_{k}(t, \xi /|\xi|)-\lambda_{k+1}(t, \xi /|\xi|)\right|+\omega(t, \xi) /|\xi|} d t \\
\leq & \int_{\Omega_{\sigma}^{k}(\xi)} \max _{1 \leq k \leq m-1} \frac{\left|\lambda_{k}^{\prime}(t, \xi /|\xi|)\right|+\left|\lambda_{k+1}^{\prime}(t, \xi /|\xi|)\right|}{0+\omega(t, \xi) /|\xi|} d t \\
& +\int_{[0, T] \backslash \Omega_{\sigma}^{k}(\xi)} \max _{1 \leq k \leq m-1} \frac{\left|\lambda_{k}^{\prime}(t, \xi /|\xi|)\right|+\left|\lambda_{k+1}^{\prime}(t, \xi /|\xi|)\right|}{\left|\lambda_{k}(t, \xi /|\xi|)-\lambda_{k+1}(t, \xi /|\xi|)\right|+0} d t \\
\leq & \max _{1 \leq k \leq m-1}\left\{\sigma^{-1} \int_{\Omega_{\sigma}^{k}(\xi)}\left(\left|\lambda_{k}^{\prime}(t, \xi /|\xi|)\right|+\left|\lambda_{k+1}^{\prime}(t, \xi /|\xi|)\right|\right) d t\right. \\
& \left.\quad+\int_{[0, T] \backslash \Omega_{\sigma}^{k}(\xi)} \frac{\left|\lambda_{k}^{\prime}(t, \xi /|\xi|)\right|+\left|\lambda_{k+1}^{\prime}(t, \xi /|\xi|)\right|}{\left|\lambda_{k}(t, \xi /|\xi|)-\lambda_{k+1}(t, \xi /|\xi|)\right|} d t\right\} \\
\leq & \max _{1 \leq k \leq m-1}\left\{\sigma^{-1} \cdot C \sigma^{1-b}+C \sigma^{-b}\right\}=C \sigma^{-b} .
\end{aligned}
$$

Noting the support of $\varphi$ and $\varphi^{\prime}$, by (2) and (23) we get

$$
\begin{aligned}
\int_{0}^{T} M_{2}(t, \xi) d t & =|\xi| \sum_{l=1}^{m-1} \int_{0}^{T} \max _{1 \leq k \leq m-1} \frac{\left|\varphi^{\prime} \cdot\left\{\lambda_{l}^{\prime}(t, \xi /|\xi|)-\lambda_{l+1}^{\prime}(t, \xi /|\xi|)\right\}\right|}{\left|\lambda_{k}(t, \xi)-\lambda_{k+1}(t, \xi)\right|+\omega(t, \xi)} d t \\
& \leq C \sigma^{-1} \sum_{l=1}^{m-1} \int_{\Omega_{2 \sigma}^{l}(\xi)}\left|\varphi^{\prime}\right| \cdot\left\{\left|\lambda_{l}^{\prime}(t, \xi /|\xi|)\right|+\left|\lambda_{l+1}^{\prime}(t, \xi /|\xi|)\right|\right\} d t \\
& \leq C \sigma^{-1} \cdot \sum_{l=1}^{m-1} C \sigma^{1-b}=C \sigma^{-b}, \\
\int_{0}^{T} M_{3}(t, \xi) d t & =\sigma|\xi| \sum_{l=1}^{m-1} \int_{0}^{T} \varphi\left(\sigma^{-1}\left\{\lambda_{l}\left(t, \frac{\xi}{|\xi|}\right)-\lambda_{l+1}\left(t, \frac{\xi}{|\xi|}\right)\right\}\right) d t \\
& =\sigma|\xi| \mu\left(\Omega_{2 \sigma}(\xi)\right) \leq \sigma|\xi| \cdot C \sigma^{a}=C \sigma^{a+1}|\xi| .
\end{aligned}
$$


By (2) and (24) we also get

$$
\begin{aligned}
\int_{0}^{T} M_{4}(t, \xi) d t \leq & \int_{0}^{T} \frac{|\xi|^{d+1-m}}{\prod_{k=1}^{m-1}\left|\lambda_{k}(t, \xi /|\xi|)-\lambda_{k+1}(t, \xi /|\xi|)\right|+\{\omega(t, \xi) /|\xi|\}^{m-1}} d t \\
\leq & \int_{\Omega_{\sigma}(\xi)} \frac{|\xi|^{d+1-m}}{0+\{\omega(t, \xi) /|\xi|\}^{m-1}} d t \\
& +\int_{[0, T] \backslash \Omega_{\sigma}(\xi)} \frac{|\xi|^{d+1-m}}{\prod_{k=1}^{m-1}\left|\lambda_{k}(t, \xi /|\xi|)-\lambda_{k+1}(t, \xi /|\xi|)\right|+0} d t \\
\leq & \sigma^{1-m}|\xi|^{d+1-m} \mu\left(\Omega_{\sigma}(\xi)\right) \\
& +|\xi|^{d+1-m} \int_{[0, T] \backslash \Omega_{\sigma}(\xi)} \frac{d t}{\prod_{k=1}^{m-1}\left|\lambda_{k}(t, \xi /|\xi|)-\lambda_{k+1}(t, \xi /|\xi|)\right|} \\
\leq & C \sigma^{a+1-m}|\xi|^{d+1-m} .
\end{aligned}
$$

Consequently, by (22), (25)-(28) it follows that

$$
\begin{aligned}
\sum_{j=0}^{m-1}|\xi|^{-j}\left|D_{t}^{j} \hat{u}(t, \xi)\right| \leq & C \sigma^{1-m} \exp \left\{C\left(\sigma^{-b}+\sigma^{a+1}|\xi|+\sigma^{a+1-m}|\xi|^{d+1-m}\right)\right\} \\
& \times\left\{\sum_{j=0}^{m-1}|\xi|^{-j}\left|\hat{u}_{j}\right|+\int_{0}^{T}|\xi|^{1-m}|\hat{f}(t, \xi)| d t\right\} .
\end{aligned}
$$

We can now conclude the proof of the theorem; notice that it is sufficient to give an estimate for $|\xi| \geq 1$, since for $|\xi| \leq 1$ we have directly from the ordinary differential equation (14) with $\xi$ as a parameter the estimate

$$
\sum_{j=0}^{m-1}\left|D_{t}^{j} \hat{u}(t, \xi)\right| \leq C(T)\left(\sum_{j=0}^{m-1}\left|\hat{u}_{j}(\xi)\right|+\int_{0}^{T}|\hat{f}(t, \xi)| d t\right) .
$$

Thus, for $|\xi| \geq 1$ choose $\sigma$ of the form $\sigma=|\xi|^{-\gamma}$ for some $\gamma>0$. Then we have

$$
\sigma^{-b}+\sigma^{a+1}|\xi|+\sigma^{a+1-m}|\xi|^{d+1-m}=|\xi|^{\gamma b}+|\xi|^{1-\gamma(a+1)}+|\xi|^{\gamma(m-a-1)+d+1-m} .
$$

The first two terms are equal if we choose

$$
\gamma=\frac{1}{a+b+1} .
$$

When

$$
d \leq \frac{m(a+b)}{a+b+1}
$$

the third term is smaller and this choice gives immediately

$$
|\xi|^{\gamma b}+|\xi|^{1-\gamma(a+1)}+|\xi|^{\gamma(m-a-1)+d+1-m} \leq 3|\xi|^{\frac{b}{a+b+1}} .
$$

Hence, by (4) there exists $\rho>0$ such that for any $(t, \xi) \in[0, T] \times \mathbf{R}_{\xi}^{n} \backslash 0$

$$
\sum_{j=0}^{m-1}|\xi|^{-j}\left|D_{t}^{j} \hat{u}(t, \xi)\right| \leq C \exp \left\{\rho|\xi|^{1 / s}\right\}\left\{\sum_{j=0}^{m-1}|\xi|^{(m-1) \gamma-j}\left|\hat{u}_{j}(\xi)\right|+\int_{0}^{T}|\xi|^{(m-1)(\gamma-1)}|\hat{f}(t, \xi)| d t\right\} .
$$

Taking into account that $u$ is a solution of (1), in virtue of Paley-Wiener theorem we find $u \in C^{m}\left([0, T] ; G^{s}\left(\mathbf{R}^{n}\right)\right)$. This concludes the proof of Theorem 1.1 in the case when $d \leq m(a+b) /(a+b+1)$. 
On the other hand, when

the dominant terms in

$$
d>\frac{m(a+b)}{a+b+1}
$$

$$
|\xi|^{\gamma b}+|\xi|^{1-\gamma(a+1)}+|\xi|^{\gamma(m-a-1)+d+1-m}
$$

are the last two (the first one is smaller). In this case we choose

$$
\gamma=\frac{m-d}{m}
$$

and proceeding as above we conclude the proof of this case.

\section{Proof of Theorem 2}

Based on the estimate (22), with either the assumptions (2) and (9) or the assumptions (3) and (10) we shall derive an a-priori estimate which shows the wellposedness in $C^{\infty}$.

We first consider the case of (2) and (9). In order to estimate $\int_{0}^{T} M_{1}(t, \xi) d t$ and $\int_{0}^{T} M_{2}(t, \xi) d t$, we need the following statement instead of Lemma 3.1.

Lemma 4.1. Assume that $\lambda_{1}, \cdots, \lambda_{m}$ belong to $A C([0, T])$ and satisfy (9). Then there exists $C>0$ such that for any $0<\sigma<1,|\xi|=1$

$$
\int_{\Omega_{\sigma}^{k}(\xi) \cup \Omega_{\sigma}^{k-1}(\xi)}\left|\lambda_{k}^{\prime}(t, \xi)\right| d t \leq C \sigma \log \sigma^{-1} .
$$

Proof. Similarly as the proof of Lemma 2, by (9) we obtain for $|\xi|=1$

$$
\begin{aligned}
\int_{\Omega_{\sigma}^{k} \cup \Omega_{\sigma}^{k-1}}\left|\lambda_{k}^{\prime}\right| d t & \leq \sum_{j=0}^{\infty} \frac{\sigma}{2^{j}} \int_{\Psi_{j}^{k}} \frac{\left|\lambda_{k}^{\prime}\right|}{\left|\lambda_{k}-\lambda_{k+1}\right|} d t+\sum_{j=0}^{\infty} \frac{\sigma}{2^{j}} \int_{\Psi_{j}^{k-1}} \frac{\left|\lambda_{k}^{\prime}\right|}{\left|\lambda_{k-1}-\lambda_{k}\right|} d t \\
& \leq 2 \sum_{j=0}^{\infty} \frac{\sigma}{2^{j}} \cdot C \log \left(\frac{\sigma}{2^{j+1}}\right)^{-1} \leq C \sigma \log \sigma^{-1} .
\end{aligned}
$$

Noting that $0 \leq d \leq m a /(a+1)$, by (2), (9) and (29) we get similarly as (25)-(28)

$$
\begin{array}{ll}
\int_{0}^{T} M_{1}(t, \xi) d t \leq C \log \sigma^{-1}, & \int_{0}^{T} M_{2}(t, \xi) d t \leq C \log \sigma^{-1} \\
\int_{0}^{T} M_{3}(t, \xi) d t \leq C \sigma^{a+1}|\xi|, & \int_{0}^{T} M_{4}(t, \xi) d t \leq C \sigma^{a+1-m}|\xi|^{\frac{a+1-m}{a+1}} .
\end{array}
$$

Therefore, by (22) it follows that

$$
\begin{aligned}
\sum_{j=0}^{m-1}|\xi|^{-j}\left|D_{t}^{j} \hat{u}(t, \xi)\right| \leq & C \sigma^{1-m} \exp \left\{C\left(\log \sigma^{-1}+\sigma^{a+1}|\xi|+\sigma^{a+1-m}|\xi|^{\frac{a+1-m}{a+1}}\right)\right\} \\
& \times\left\{\sum_{j=0}^{m-1}|\xi|^{-j}\left|\hat{u}_{j}(\xi)\right|+\int_{0}^{T}|\xi|^{1-m}|\hat{f}(t, \xi)| d t\right\} .
\end{aligned}
$$


We next consider also the case of (3) and (10) (see Remark 1.9). Noting that $0 \leq d \leq m-1$ and (2) also holds with $a=m-1$, by (3), (10) and (23) we get similarly as (25)-(28)

$$
\begin{array}{ll}
\int_{0}^{T} M_{1}(t, \xi) d t \leq C(2 \sigma)^{-b}, & \int_{0}^{T} M_{2}(t, \xi) d t \leq C(2 \sigma)^{-b}, \\
\int_{0}^{T} M_{3}(t, \xi) d t \leq C \sigma \exp \left\{-(2 \sigma)^{-b}\right\}|\xi|, & \int_{0}^{T} M_{4}(t, \xi) d t \leq C .
\end{array}
$$

Therefore, by (22) it follows that

$$
\begin{aligned}
\sum_{j=0}^{m-1}|\xi|^{-j}\left|D_{t}^{j} \hat{u}(t, \xi)\right| \leq & C \sigma^{1-m} \exp \left[C\left\{(2 \sigma)^{-b}+\sigma \exp \left\{-(2 \sigma)^{-b}\right\}|\xi|+1\right\}\right] \\
& \times\left\{\sum_{j=0}^{m-1}|\xi|^{-j}\left|\hat{u}_{j}(\xi)\right|+\int_{0}^{T}|\xi|^{1-m}|\hat{f}(t, \xi)| d t\right\} .
\end{aligned}
$$

For $|\xi| \geq e$ choose $\sigma=|\xi|^{-1 /(a+1)}$ in (30) and $2 \sigma=(\log |\xi|)^{-1 / b}$ in (31). Then there exists $\rho>0$ such that for any $(t, \xi) \in[0, T] \times \mathbf{R}_{\xi}^{n} \backslash 0$

$$
\sum_{j=0}^{m-1}|\xi|^{-j}\left|D_{t}^{j} \hat{u}(t, \xi)\right| \leq C|\xi|^{-\rho}\left\{\sum_{j=0}^{m-1}|\xi|^{-j}\left|\hat{u}_{j}(\xi)\right|+\int_{0}^{T}|\xi|^{1-m}|\hat{f}(t, \xi)| d t\right\} .
$$

The Cauchy problem (1) has a cone of dependence (see [13], [15], [23]). Thus, taking into account that $u$ is a solution of (1), in virtue of Paley-Wiener theorem we find $u \in C^{m}\left([0, T] ; C^{\infty}\left(\mathbf{R}^{n}\right)\right)$. This concludes the proof of Theorem 1.8.

\section{Appendix}

In order to apply Theorem 1.1 to Example 1.15, we shall check the assumptions (2) and (3). For the simplicity, it is enough to consider the second order polynomial $P(t, \tau, \xi) \equiv \tau^{2}-t^{2 h} \sin ^{2 h}\left(1 / t^{h-1}\right) \cdot \xi^{2}$ where $h$ is a even number, and put

$$
\lambda_{1}(t)=t^{h} \sin ^{h}\left(\frac{1}{t^{h-1}}\right) \text { and } \lambda_{2}(t)=-t^{h} \sin ^{h}\left(\frac{1}{t^{h-1}}\right) .
$$

Check of the condition (2). Since $\sin ^{h} \theta=\sin ^{h}(\theta-j \pi) \geq(2 / \pi)^{h}(\theta-j \pi)^{h}$ for $j \pi-\pi / 2 \leq \theta \leq j \pi+\pi / 2$, we obtain for $t \in\left[(j \pi+\pi / 2)^{-1 /(h-1)},(j \pi-\pi / 2)^{-1 /(h-1)}\right]$

$$
\lambda_{1}(t) \equiv t^{h} \sin ^{h}\left(\frac{1}{t^{h-1}}\right) \geq\left(\frac{2}{\pi}\right)^{h} t^{h}\left(\frac{1}{t^{h-1}}-j \pi\right)^{h} .
$$

The equation $\sigma=t^{h} \sin ^{h}\left(1 / t^{h-1}\right)$ has two distinct roots $\alpha_{j}$ and $\beta_{j}\left(\alpha_{j}<\beta_{j}\right)$ in the interval $\left[(j \pi+\pi / 2)^{-1 /(h-1)},(j \pi-\pi / 2)^{-1 /(h-1)}\right]$. While, the equation $\sigma=(2 / \pi)^{h} t^{h}\left(1 / t^{h-1}-j \pi\right)^{h}$ has also two distinct roots $\tilde{\alpha}_{j}$ and $\tilde{\beta}_{j}\left(\tilde{\alpha}_{j}<\tilde{\beta}_{j}\right)$ in the interval $\left[(j \pi+\pi / 2)^{-1 /(h-1)},(j \pi-\pi / 2)^{-1 /(h-1)}\right]$ which satisfy $\tilde{\beta}_{j}-\tilde{\alpha}_{j} \leq \sigma^{1 / h} j^{-1}$. Therefore by (32) it follows that

$$
\beta_{j}-\alpha_{j} \leq \tilde{\beta}_{j}-\tilde{\alpha}_{j} \leq \frac{\sigma^{\frac{1}{h}}}{j} .
$$

Let $J$ be the number satisfying $\{(J+1) \pi+\pi / 2\}^{-h /(h-1)} \leq \sigma \leq(J \pi+\pi / 2)^{-h /(h-1)}$. Hence, there exist $C$ and $C^{\prime}>0$ such that

$$
C \sigma^{-1+\frac{1}{h}} \leq J \leq C^{\prime} \sigma^{-1+\frac{1}{h}}
$$


Thus by (33) and (34) we get

$\mu\left(\Omega_{2 \sigma}\right) \leq \frac{1}{(J \pi+\pi / 2)^{\frac{1}{h-1}}}+\sum_{j=1}^{J}\left(\beta_{j}-\alpha_{j}\right) \leq C \sigma^{\frac{1}{h}}+\sum_{j=1}^{C^{\prime} \sigma^{-1+1 / h}} \frac{\sigma^{\frac{1}{h}}}{j} \leq C \sigma^{\frac{1}{h}} \log \sigma^{-1}$.

Changing $2 \sigma$ into $\sigma$, we find that $\mu\left(\Omega_{\sigma}\right) \leq C \sigma^{a}$ for $a<1 / h$.

Check of the condition (3). Let $\left\{\gamma_{j}\right\}$ be the sequence satisfying

$$
\lambda_{1}^{\prime}\left(\gamma_{j}\right)=0 \text { and } \alpha_{j}>\gamma_{j}>\frac{1}{(j \pi+\pi / 2)^{\frac{1}{h-1}}}>\beta_{j+1} \text { for any } j=1,2, \cdots .
$$

We remark that there exists $c>0$ such that $c \leq \sin ^{2} 1 / \gamma_{j}^{h-1} \leq 1$ for any $j=$ $1,2, \cdots$. Thus by (34) and (35) we get

$$
\begin{aligned}
& \int_{[0, T] \backslash \Omega_{2 \sigma}} \frac{\left|\lambda_{1}^{\prime}\right|+\left|\lambda_{2}^{\prime}\right|}{\left|\lambda_{1}-\lambda_{2}\right|} d t=\sum_{j=1}^{J} \int_{\beta_{j+1}}^{\alpha_{j}} \frac{\left|\left\{t^{h} \sin ^{h}\left(\frac{1}{t^{h-1}}\right)\right\}^{\prime}\right|}{2 t^{h} \sin ^{h}\left(\frac{1}{t^{h-1}}\right)} d t \\
= & \frac{1}{2} \sum_{j=1}^{J} \log \frac{\gamma_{j}^{h} \sin ^{h}\left(\frac{1}{\gamma_{j}^{h-1}}\right)}{\beta_{j+1}^{h} \sin ^{h}\left(\frac{1}{\beta_{j+1}^{h-1}}\right)} \cdot \frac{\gamma_{j}^{h} \sin ^{h}\left(\frac{1}{\gamma_{j}^{h-1}}\right)}{\alpha_{j}^{h} \sin ^{h}\left(\frac{1}{\alpha_{j}^{h-1}}\right)}=\frac{1}{2} \sum_{j=1}^{J} \log \frac{\gamma_{j}^{2 h} \sin ^{2 h}\left(\frac{1}{\gamma_{j}^{h-1}}\right)}{\sigma^{2}} \\
\leq & C \sum_{j=1}^{J} \log \gamma_{j}+C J \log \sigma^{-1} \leq C \sum_{j=1}^{C^{\prime} \sigma^{-1+1 / h}} \log \frac{1}{j}+C \sigma^{-1+1 / h} \log \sigma^{-1} \\
\leq & C \sigma^{-1+1 / h} \log \sigma^{-1} .
\end{aligned}
$$

Changing $2 \sigma$ into $\sigma$, we find that $\int_{[0, T] \backslash \Omega_{\sigma}} \frac{\left|\lambda_{1}^{\prime}\right|+\left|\lambda_{2}^{\prime}\right|}{\left|\lambda_{1}-\lambda_{2}\right|} d t \leq C \sigma^{-b}$ for $b>1-1 / h$.

\section{REFERENCES}

[1] M.D. Bronštein, The Cauchy problem for hyperbolic operators with characteristics of variable multiplicity, Trudy Moskov. Mat. Obšc̆ 41, 87-103 (1980)(Trans. Moscow Math. Soc. 1, 87$103(1982))$.

[2] M. Cicognani, On the strictly hyperbolic equations which are Hölder continuous with respect to time, Ital. J. Pure Appl. Math. 4, 73-82 (1988).

[3] F. Colombini, E. De Giorgi and S. Spagnolo, Sur les équations hyperboliques avec des coefficients qui ne dépendent que du temps, Ann. Scuola Norm Sup. Pisa 6, 511-559 (1979).

[4] F. Colombini, H. Ishida, Well-posedness of the Cauchy problem in Gevrey classes for some weakly hyperbolic equations of higher order, to appear.

[5] F. Colombini, E. Jannelli and S. Spagnolo, Wellposedness in the Gevrey classes of the Cauchy problem for a non strictly hyperbolic equation with coefficients depending on time, Ann. Scuola Norm Sup. Pisa 10, 291-312 (1983).

[6] F. Colombini, T. Kinoshita, On the Gevrey wellposedness of the Cauchy problem for weakly hyperbolic equations of higher order, J. Differential Equations 186, 394-419 (2002).

[7] F. Colombini, N. Orrú, Well posedness in $C^{\infty}$ for some weakly hyperbolic equations, J. Math. Kyoto. Univ. 39, 399-420 (1999).

[8] P. D'Ancona, Gevrey well posedness of an abstract Cauchy problem of weakly hyperbolic type, Publ. RIMS Kyoto Univ. 24, 433-449 (1988).

[9] P. D'Ancona, Well posedness in $C^{\infty}$ for a weakly hyperbolic second order equation, Rend. Sem. Mat. Univ. Padova 91, 65-83 (1994).

[10] T. Gramchev and L. Rodino, Gevrey solvability for semilinear partial differential equations with multiple characteristics, Boll. Unione Mat. Ital. Sez. B Artic. Ric. Mat. (8) 2, 65-120 (1999).

[11] H. Ishida and K. Yagdjian, preprint.

[12] V. Ya. Ivrii, Cauchy problem conditions for hyperbolic operators with characteristics of variable multiplicity for Gevrey classes, Siberian. Math. 17, 921-931 (1976). 
[13] K. Kajitani, S. Wakabayashi and K. Yagdjian, The $C^{\infty}$-well posed Cauchy problem for hyperbolic operators with multiple characteristics vanishing with the different speeds, Osaka J. Math. 39, 447-485 (2002).

[14] T. Mandai, Smoothness of roots of hyperbolic polynomials with respect to one-dimensional parameter, Bull. Fac. Gen. Ed. Gifu Univ. 21, 115-118 (1985).

[15] S. Mizohata, Theory of partial differential equations, Cambridge university press, Cambridge (1973).

[16] T. Nishitani, The Cauchy problem for weakly hyperbolic equations of second order, Comm. P. D. E. 5, 1273-1296 (1980).

[17] T. Nishitani, Sur les équations hyperboliques à coefficients hölderiens en $t$ et de classes de Gevrey en $x$, Bull. Sci. Math. 107, 113-138 (1983).

[18] Y. Ohya and S. Tarama, Le problème de Cauchy àcaractéristiques multiples -coefficients hölderiens en t-, Hyperbolic equations and related topics (Katata/Kyoto, 1984), 273-306, Academic Press, Boston, MA, 1986.

[19] M. Reissig and K. Yagdjian, Weakly hyperbolic equation with fast oscillating coefficients, Osaka J. Math. 36, 437-464 (1999).

[20] S. Spagnolo, On the Absolute Continuity of the Roots of Some Algebraic Equations, Ann. Univ.Ferrara - Sez. VII - Sc. Mat. Suppl. XLV, 327-337 (1999).

[21] S. Tarama, On the second order hyperbolic equations degenerating in the infinite order. -Example-, Math. Japonica 42, 523-534 (1995).

[22] S. Wakabayashi, Remarks on hyperbolic polynomials, Tsukuba J. Math. 10, 17-28 (1986).

[23] K. Yagdjian, The Cauchy problem for hyperbolic operators. Multiple characteristics. Microlocal approach, Mathematical Topics, 12, Akademie Verlag, Berlin (1997).

Addresses of the authors

Piero D'Ancona, Università "La Sapienza" di Roma, Piazzale Aldo Moro, 2, I-00185 Roma, Italy

e-mail: dancona@mat.uniroma1.it

Tamotu Kinoshita, Institute of Mathematics, Tsukuba University, Tsukuba Ibaraki 305-8571, Japan

e-mail: kinosita@math.tsukuba.ac.jp 